\title{
Hospital Pharmacist Workforce in Eleven-Year 2006-2016 at Ministry of Health in Saudi Arabia
}

\author{
Yousef Ahmed Alomi ${ }^{1 *}$, Saeed Jamaan Alghamdi², Radi Abdullah Alattyh ${ }^{2}$ \\ ${ }^{1}$ The Past General Manager of General Administration of Pharmaceutical Care and The Past Head, National Clinical pharmacy, \\ and pharmacy practice and Pharmacy $R$ and D Administration, Ministry of Health, Riyadh, KSA. \\ ${ }^{2}$ General Administration of Pharmaceutical Care, Ministry of Health, P.O.BOX 100, Riyadh 11392, Riyadh, KSA.
}

Received: 13 December 2017;

Accepted: 28 February 2018

*Correspondence to:

Dr. Yousef Ahmed Alomi, The Past General Manager of General Administration of Pharmaceutical Care, The Past Head, National Clinical pharmacy, and pharmacy practice, Head, Pharmacy $R$ and $D$ Administration, Ministry of Health, P.O.BOX 100, Riyadh 11392, Riyadh, Saudi Arabia.. Email:yalomi@gmail.com

Copyright: () the author(s),publisher and licensee Indian Academy of Pharmacists. This is an open-access article distributed under the terms of the Creative Commons Attribution Non-Commercial License, which permits unrestricted non-commercial use, distribution, and reproduction in any medium, provided the original work is properly cited.

\begin{abstract}
Objective: The workforce of hospital pharmacist is one the elements of pharmacy strategic plan in Saudi Arabia. The purpose of this study is to explore hospital pharmacist's workforce in eleven years (2006-2016) at Ministry of Health in Saudi Arabia. Methods: It is a retrospective analysis of eleven years (2006-2016) of $\mathrm{MOH}$ hospital pharmacist workforce. All data were derived from Ministry of Health statistical yearbooks. It included pharmacist or clinical pharmacist's workforces at $\mathrm{MOH}$ hospitals. The type of hospital included having bed capacity of 50-1500 beds with Adults public hospitals, pediatrics and maternity hospitals, Psychiatric hospitals, Diabetic centers, Cardiology centers, Oncology Centers, Renal Dialysis Centers, and Medical cities. All calculations were based on $\mathrm{MOH}$ workforce standards of hospitals. The calculations were per each region for a total of twenty-one regions; the hospital's distribution numbers, the pharmacist distribution numbers, the ratio of pharmacist per hospital, the gender distribution of Pharmacist per region, the gender distribution of Saudi pharmacist per each region across Saudi Arabia. Result: The total numbers of hospitals were 276 hospitals, 2,325 primary care centers, and 20 administration regions. The total numbers of pharmacists were 3,520, distributed at hospital 2,760 (78.41\%), at primary care centers 430 $(12.2 \%)$ while administration regions 330 (9.37\%). The average numbers of hospitals were (251.36) with a range number of (220-274), and the total number was (274). The average numbers of hospital pharmacists were $(1,617.55)$ with a range number of $(636-2760)$, and the total number was $(2760)$. The total gender distribution of all hospital pharmacists was male 1,077.4 (61.30\%) and female 680.1 (38.70\%). The total nationality distribution of all hospital pharmacists was Saudi $1342.03(81.57 \%)$ and non-Saudi 303.26 (18.43\%(. Of Saudi primary care center pharmacist 861.05 (64.16\%) was male and 480.98 (35.84\%) was female. Conclusion: Number hospitals are increased over the past years. Despite the incremental number of hospital but the pharmacists over past years are same. Most of the hospital pharmacists were Saudi with more of half of staff were males. Targeting of the increasing number of Saudi hospital pharmacists through complete Saudisation standard is required.
\end{abstract}

Key words: Hospital pharmacist, Workforce, Manpower, Ministry of health, Saudi arabia.

\section{INTRODUCTION}

The pharmaceutical care founded in the early 2000 s by the American society of health system pharmacists (ASHP). ${ }^{[1]}$ The major pillar of the concept was a pharmacist. During the implementation of the concept required enough number of a pharmacist and pharmacy qualification. ${ }^{[2]}$ The ASHP and other pharmacy organizations periodically published several investigations about the pharmacy workforce resources. It includes a pharmacist, clinical pharmacy and pharmacy technician in the United States of America (USA) and other countries. ${ }^{[3-14]}$ The number required is increased over the years. The world health organization has Published pharmacy workforce in Middle East countries with comparable among them and including Saudi Arabia. ${ }^{[9]}$ The report did discuss detail about nationality and gender distribution among cities at each country per each country, and even the report did not mention the detail about clinical pharmacy or pharmacy technicians. The authors are not familiar with any study in Saudi Arabia or Middle East countries to investigate the hospital pharmacist workforce. The goal of this study is to explore the pharmacist workforce over the past eleven years (2006-2016) at $\mathrm{MOH}$ hospitals in Kingdom of Saudi Arabia.

\section{METHODS}

It is a retrospective analysis of eleven years (2006-2016) of MOH hospital pharmacist workforce. All data were derived from Ministry of Health statistical yearbooks. ${ }^{[15-25]}$ It included pharmacist or clinical pharmacist's workforces at $\mathrm{MOH}$ hospitals. The type of hospital included having bed capacity 50-1500 beds with Adults public hospitals, pediatrics and maternity hospitals, Psychiatric hospitals, Diabetic centers, Cardiology centers, Oncology Centers, Renal Dialysis Centers, and Medical cities. All pharmacists were expected to provide pharmaceutical according to ASHP definition and requirements. ${ }^{[1-2]}$ All type of pharmacy services were based on Saudi Central Board of hospital accreditation, Joint commission of hospital accreditation, ASHP best practice standards, and general administration of the pharmaceutical care strategic plan. ${ }^{[26-28]}$ All Pharmacist works at $\mathrm{MOH}$ administration or non-MOH government hospitals and primary care centers were excluded from the studies. All private hospitals or community pharmacists were excluded from the study. All pharmacy technicians were excluded from the study. All calculations were based on $\mathrm{MOH}$ workforce standards of hospitals. The calculations were per each region for a total twenty-one regions; the hospital's distribution numbers, the pharmacist 
distribution numbers, the ratio of pharmacist per hospital, the gender distribution of Pharmacist per region and the gender distribution of Saudi Pharmacist per each region across Saudi Arabia. All calculation was done used Microsoft Excel version ten.

\section{RESULT}

The total numbers of hospitals were 276 hospitals, 2,325 primary care centers, and 20 administration regions. The total numbers of small hospitals were $140(50.72 \%)$, medium hospitals 95 (34.42\%), small hospitals 18 (6.52\%), Large hospitals $21(7.61 \%)$, and medical cities $2(0.72 \%)$. The total numbers of pharmacists were 3,520, distributed at hospital 2,760 (78.41\%), at primary care centers $430(12.2 \%)$ while administration regions $330(9.37$ $\%)$ the last update information October 2016 as explored in Table 1. The average numbers of hospitals were (251.36) with a range number of (220274), and the total number was (274). The highest region had a number of hospitals; Riyadh (44.45) followed by East Province (19.27) and Madina regions (19.09). The average numbers of hospital pharmacists were $(1,617.55)$ with a range number of (636-2760), and the total number was (2760). The region had highest an average number of hospital pharmacists were Riyadh (392.64) followed by East Province (149.36) and Makkah regions (146.09) pharmacists as explore in Table 2, 3. The total gender distribution of all hospital pharmacists was male 1,077.4 (61.30\%) and female $680.1(38.70 \%)$. The total nationality distribution of all hospital pharmacists was Saudi $1342.03(81.57 \%)$ and non-Saudi 303.26 (18.43\%(. Of Saudi primary care center pharmacist $861.05(64.16 \%)$ were male and $480.98(35.84 \%)$ were female as explored in Table 4, 5 and 6.

\section{DISCUSSION}

The Ministry of Health in Kingdom of Saudi Arabia founded strategic health planning for ten years. ${ }^{[29]}$ The plan consisted of several elements including Human Resources of healthcare professionals. The pharmacy administration at $\mathrm{MOH}$ emphasized the element by establishment of pharmacy strategic plan with six general goals and pharmacy workforce was part of it. ${ }^{[28]}$ Before 2006, the numbers of pharmacists per each pharmacy organization including hospitals and primary care centers were not well established. The first $\mathrm{MOH}$ statistical book have reported the number of a pharmacists. ${ }^{[15]}$ In mid of the 2000s, the pharmacy administration established the requirements needed for hospital pharmacy staff. ${ }^{[30-31]}$ The new guidelines updated the hospital pharmacy staff requirements released during pharmacy strategic plan. ${ }^{[28]}$ The numbers increased several times, but the question rose what was the pharmacy workforce over past ten years, did the increments corrected, based on what's the decision made. The author explored the analysis of past eleven years the pharmacy staff at Ministry of Health hospitals. The findings showed that there was a moderate increase in the number of hospitals while the number of pharmacists did not increase accordingly. The number of graduated pharmacists were low; while the number has increased by establishment of several colleges of pharmacies. ${ }^{[25]}$ The gender distribution found the male pharmacist little bit more than female pharmacists with increased number over the past years. The study results almost similar that earlier reported elsewhere. ${ }^{[8,12]}$ While is lower than in female pharmacist what reported by Gal, Diane et al. in Europe, Western Pacific, Eastern Mediterranean countries without apparent reasons. ${ }^{[9]}$ The number of the pharmacists were lower that's what found by Mcleod, Donald C, and study

\begin{tabular}{|c|c|c|c|c|c|c|c|}
\hline & Region & No of Hospitals & $\begin{array}{l}\text { Small } \\
<100\end{array}$ & $\begin{array}{l}\text { Medium } \\
100-299\end{array}$ & $\begin{array}{l}\text { Large } \\
300-399\end{array}$ & $\begin{array}{l}\text { Large } \\
400->\text { or }=600\end{array}$ & Medical Cities \\
\hline 1 & Riyadh & 42 & 19 & 19 & 1 & 2 & 1 \\
\hline 2 & Makkah & 10 & 3 & 2 & 2 & 2 & 1 \\
\hline 3 & Jeddah & 13 & 2 & 7 & 1 & 3 & 0 \\
\hline 4 & Taif & 13 & 9 & 1 & 0 & 3 & 0 \\
\hline 5 & Madina & 20 & 12 & 6 & 0 & 2 & 0 \\
\hline 6 & Quseen & 19 & 10 & 6 & 1 & 2 & 0 \\
\hline 7 & East Province & 20 & 13 & 2 & 3 & 2 & 0 \\
\hline 8 & Alhasa & 11 & 4 & 5 & 0 & 2 & 0 \\
\hline 9 & Heralbatin & 7 & 4 & 1 & 2 & 0 & 0 \\
\hline 10 & Aseer & 20 & 8 & 11 & 0 & 1 & 0 \\
\hline 11 & Bisha & 7 & 4 & 2 & 1 & 0 & 0 \\
\hline 12 & Tabouk & 11 & 3 & 7 & 0 & 1 & 0 \\
\hline 13 & Hail & 13 & 10 & 3 & 0 & 0 & 0 \\
\hline 14 & North Boarder & 9 & 3 & 4 & 2 & 0 & 0 \\
\hline 15 & Jazan & 21 & 13 & 7 & 0 & 1 & 0 \\
\hline 16 & Najran & 12 & 8 & 3 & 1 & 0 & 0 \\
\hline 17 & Albaha & 10 & 7 & 1 & 2 & 0 & 0 \\
\hline 18 & Aljouf & 9 & 3 & 4 & 2 & 0 & 0 \\
\hline 19 & Alqurayat & 4 & 2 & 2 & 0 & 0 & 0 \\
\hline \multirow[t]{2}{*}{20} & Qunfetha & 5 & 3 & 2 & 0 & 0 & 0 \\
\hline & Total & 276 & 140 & 95 & 18 & 21 & 2 \\
\hline
\end{tabular}


Table 2: Total number of hospitals.

\begin{tabular}{|c|c|c|c|c|c|c|c|c|c|c|c|c|}
\hline & 2006 & 2007 & 2008 & 2009 & 2010 & 2011 & 2012 & 2013 & 2014 & 2015 & 2016 & Average \\
\hline Riyadh & 40 & 40 & 42 & 44 & 44 & 45 & 46 & 47 & 47 & 47 & 47 & 44.45 \\
\hline Makkah & 8 & 8 & 9 & 10 & 10 & 10 & 37 & 10 & 10 & 10 & 10 & 12.00 \\
\hline Jeddah & 12 & 12 & 12 & 12 & 12 & 12 & Missing Data & 13 & 13 & 14 & 13 & 12.50 \\
\hline Taif & 11 & 11 & 11 & 12 & 12 & 13 & Missing Data & 14 & 14 & 13 & 13 & 12.40 \\
\hline Madina & 16 & 17 & 17 & 20 & 20 & 20 & 20 & 20 & 20 & 20 & 20 & 19.09 \\
\hline Quseen & 16 & 16 & 17 & 17 & 17 & 17 & 18 & 18 & 18 & 18 & 18 & 17.27 \\
\hline East Province & 17 & 18 & 18 & 19 & 18 & 18 & 32 & 18 & 18 & 18 & 18 & 19.27 \\
\hline Alhasa & 8 & 8 & 8 & 9 & 9 & 9 & Missing Data & 9 & 10 & 10 & 10 & 9.00 \\
\hline Heralbatin & 4 & 4 & 4 & 5 & 5 & 5 & Missing Data & 7 & 7 & 7 & 7 & 5.50 \\
\hline Aseer & 18 & 18 & 19 & 16 & 16 & 16 & 27 & 21 & 20 & 20 & 20 & 19.18 \\
\hline Bisha & 3 & 3 & 3 & 7 & 7 & 7 & Missing Data & 7 & 7 & 7 & 7 & 5.80 \\
\hline Tabouk & 10 & 10 & 10 & 11 & 11 & 11 & 11 & 11 & 11 & 11 & 11 & 10.73 \\
\hline Hail & 9 & 9 & 9 & 9 & 11 & 11 & 11 & 11 & 12 & 12 & 12 & 10.55 \\
\hline North Boarder & 6 & 7 & 7 & 7 & 7 & 7 & 7 & 8 & 8 & 9 & 9 & 7.45 \\
\hline Jazan & 15 & 15 & 15 & 16 & 18 & 18 & 19 & 20 & 21 & 21 & 21 & 18.09 \\
\hline Najran & 10 & 9 & 9 & 9 & 11 & 11 & 10 & 10 & 10 & 11 & 11 & 10.09 \\
\hline Albaha & 9 & 10 & 10 & 10 & 10 & 10 & 10 & 11 & 10 & 10 & 10 & 10.00 \\
\hline Aljouf & 5 & 6 & 7 & 6 & 6 & 6 & 11 & 7 & 8 & 8 & 9 & 7.18 \\
\hline Alqurayat & 2 & 3 & 3 & 4 & 3 & 3 & Missing Data & 4 & 4 & 4 & 4 & 3.40 \\
\hline Qunfetha & 1 & 1 & 1 & 1 & 2 & 2 & Missing Data & 2 & 2 & 4 & 4 & 2.00 \\
\hline TOTAL & 220 & 225 & 231 & 244 & 249 & 251 & 259 & 268 & 270 & 274 & 274 & 251.36 \\
\hline Average per city & 11.000 & 11.250 & 11.550 & 12.200 & 12.450 & 12.550 & 19.923 & 13.400 & 13.500 & 13.700 & 13.700 & 13.20 \\
\hline
\end{tabular}

Table 3: Total number of hospital pharmacist.

\begin{tabular}{|c|c|c|c|c|c|c|c|c|c|c|c|c|}
\hline & 2006 & 2007 & 2008 & 2009 & 2010 & 2011 & 2012 & 2013 & 2014 & 2015 & 2016 & Average \\
\hline Riyadh & 137 & 243 & 326 & 333 & 367 & 404 & 394 & 439 & 497 & 504 & 675 & 392.64 \\
\hline Makkah & 62 & 60 & 69 & 91 & 105 & 75 & 309 & 121 & 230 & 241 & 244 & 146.09 \\
\hline Jeddah & 48 & 39 & 75 & 104 & 110 & 102 & Missing Data & 105 & 142 & 181 & 191 & 109.70 \\
\hline Taif & 30 & 27 & 35 & 42 & 53 & 50 & Missing Data & 82 & 90 & 115 & 118 & 64.20 \\
\hline Madina & 35 & 38 & 43 & 86 & 94 & 83 & 100 & 118 & 137 & 137 & 149 & 92.73 \\
\hline Quseen & 38 & 59 & 75 & 82 & 108 & 97 & 83 & 114 & 136 & 135 & 163 & 99.09 \\
\hline East Province & 50 & 86 & 92 & 109 & 156 & 153 & 276 & 164 & 169 & 191 & 197 & 149.36 \\
\hline Alhasa & 42 & 27 & 34 & 46 & 59 & 54 & Missing Data & 57 & 62 & 82 & 91 & 55.40 \\
\hline Heralbatin & 7 & 8 & 11 & 26 & 32 & 26 & Missing Data & 26 & 32 & 35 & 46 & 24.90 \\
\hline Aseer & 20 & 41 & 53 & 72 & 111 & 110 & 164 & 154 & 163 & 181 & 190 & 114.45 \\
\hline Bisha & 12 & 9 & 21 & 13 & 20 & 19 & Missing Data & 25 & 42 & 28 & 40 & 22.90 \\
\hline Tabouk & 20 & 26 & 28 & 44 & 37 & 29 & 35 & 53 & 66 & 76 & 99 & 46.64 \\
\hline Hail & 28 & 24 & 27 & 31 & 34 & 23 & 25 & 38 & 53 & 58 & 65 & 36.91 \\
\hline North Boarder & 6 & 7 & 13 & 39 & 37 & 30 & 28 & 37 & 40 & 40 & 61 & 30.73 \\
\hline Jazan & 41 & 44 & 50 & 93 & 114 & 95 & 104 & 106 & 128 & 135 & 151 & 96.45 \\
\hline Najran & 7 & 28 & 52 & 53 & 69 & 56 & 59 & 64 & 79 & 99 & 111 & 61.55 \\
\hline Albaha & 23 & 10 & 30 & 47 & 40 & 35 & 42 & 53 & 56 & 52 & 63 & 41.00 \\
\hline Aljouf & 17 & 18 & 18 & 31 & 33 & 26 & 42 & 31 & 38 & 50 & 54 & 32.55 \\
\hline Alqurayat & 11 & 8 & 6 & 18 & 15 & 12 & Missing Data & 18 & 22 & 25 & 28 & 16.30 \\
\hline Qunfetha & 2 & 2 & 7 & 4 & 7 & 8 & Missing Data & 15 & 24 & 24 & 24 & 11.70 \\
\hline TOTAL & 636 & 804 & 1065 & 1364 & 1601 & 1487 & 1661 & 1820 & 2206 & 2389 & 2760 & $1,617.55$ \\
\hline Average per city & 31.8 & 40.2 & 53.25 & 68.2 & 80.05 & 74.35 & 127.7692 & 91 & 110.3 & 119.45 & 138 & 84.94 \\
\hline
\end{tabular}




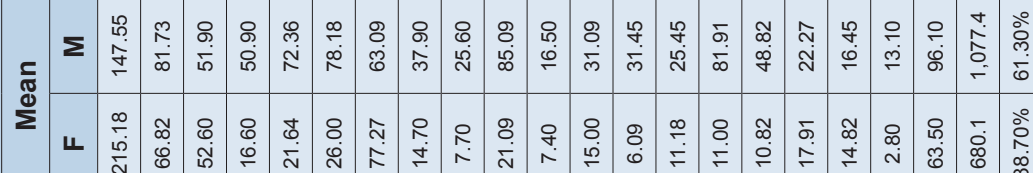

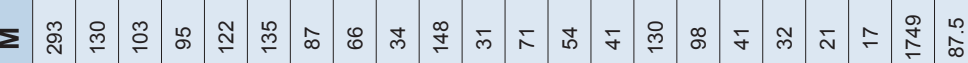
$\stackrel{0}{\circ}$

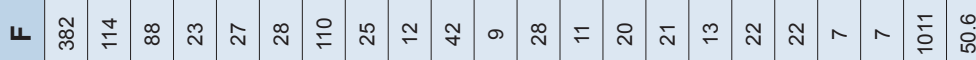
ก

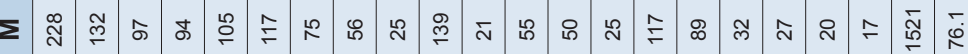

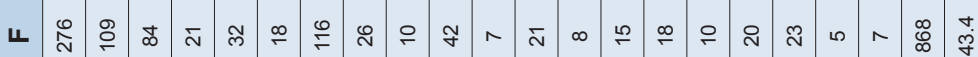
芦

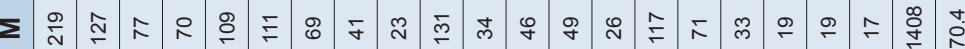

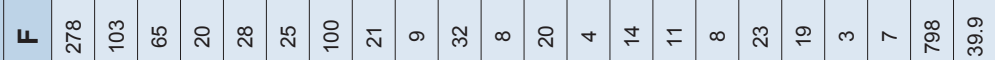
กั

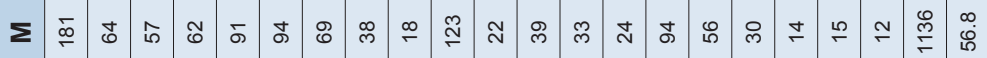

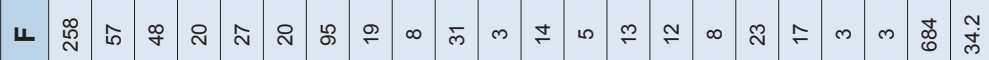

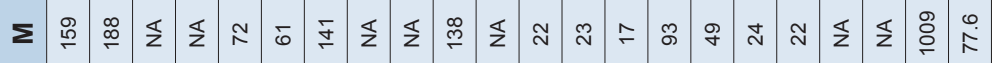
กั

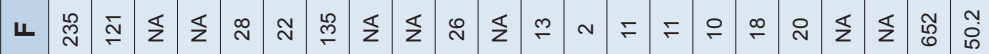
זัฐ ஜ

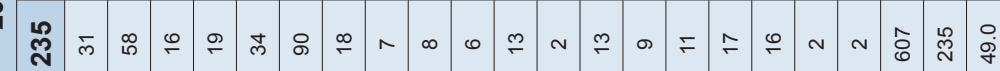
울

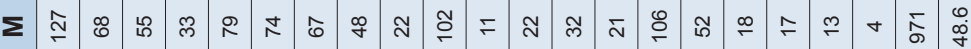

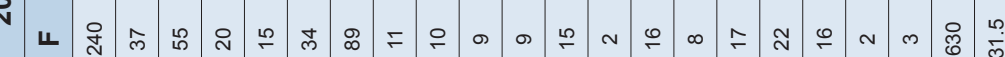

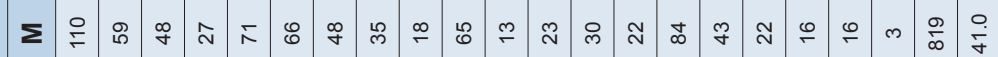

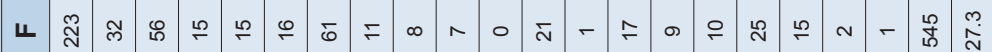

ঠ்

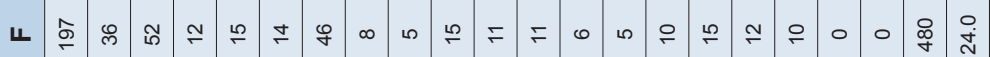

$\sum$ \&

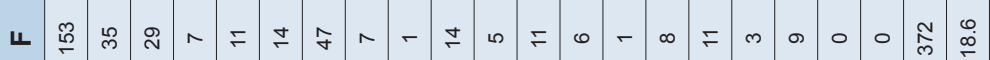

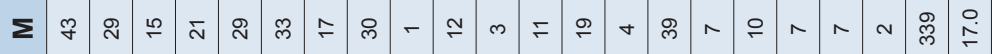

ᄂ ப

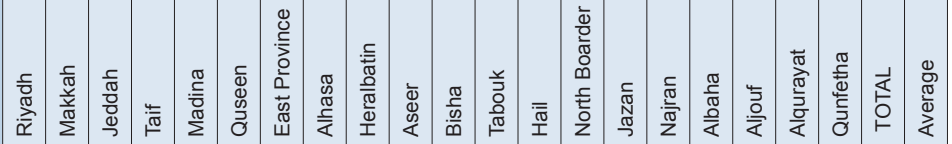

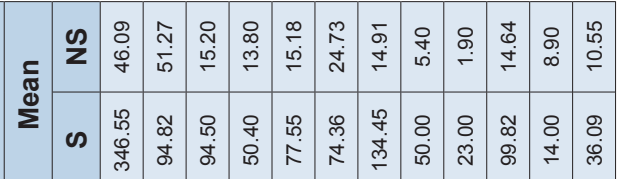

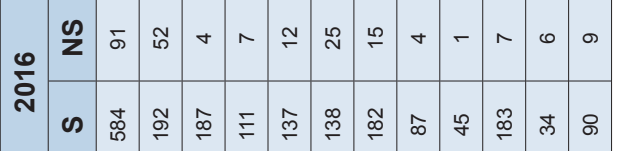

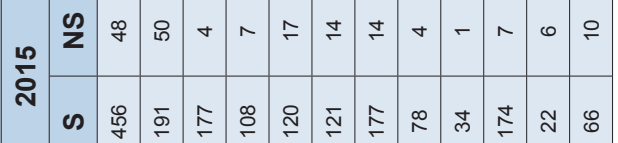

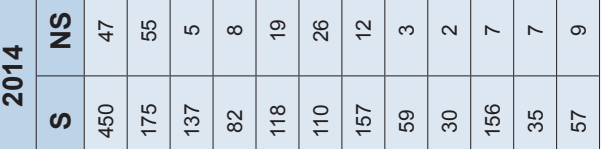

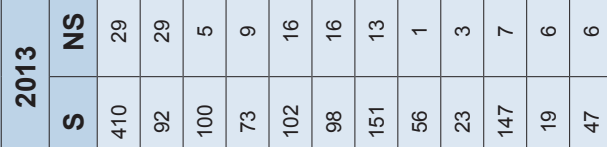

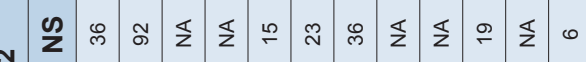

ํํำ

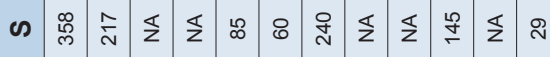

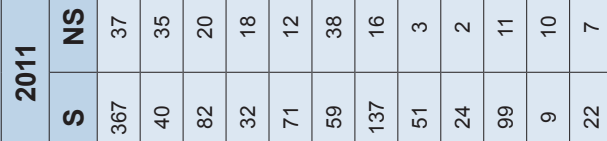

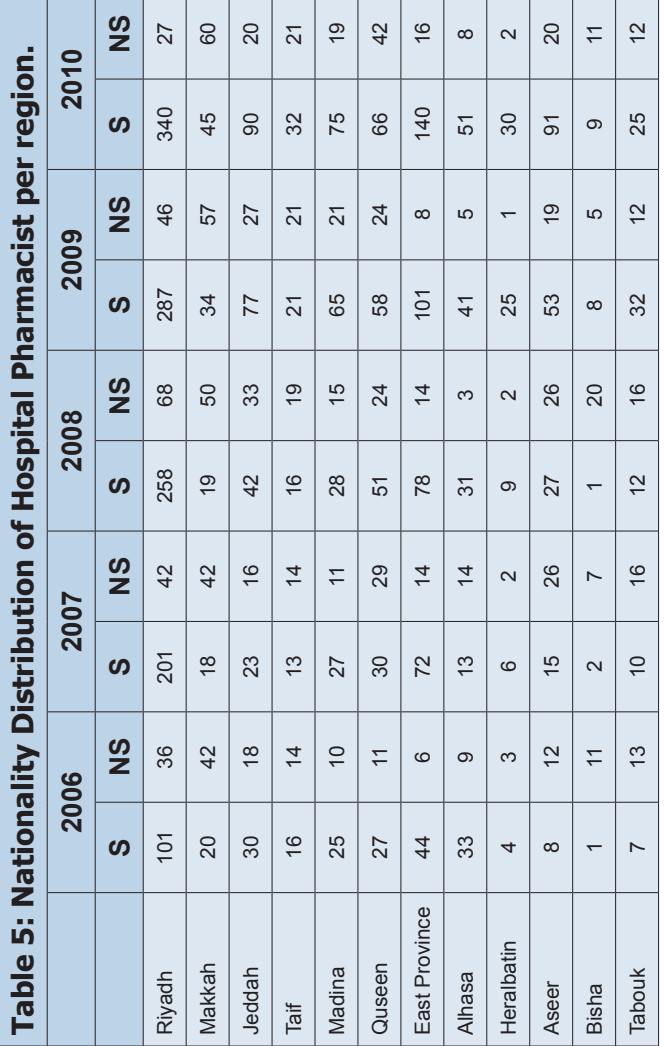




\begin{tabular}{|c|c|c|c|c|c|c|c|c|c|}
\hline & 品 & $\begin{array}{l}\bar{\delta} \\
\stackrel{\delta}{\rho}\end{array}$ & $\stackrel{\stackrel{\sim}{\infty}}{\stackrel{\leftrightarrow}{\rightleftharpoons}}$ & 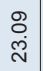 & $\stackrel{\infty}{\stackrel{\infty}{\sim}}$ & $\underset{\mathrm{i}}{\stackrel{\text { O}}{ }}$ & & 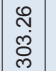 & \begin{tabular}{l}
$\stackrel{\circ}{\circ}$ \\
\multirow{\infty}{\infty}{} \\
$\stackrel{\infty}{2}$
\end{tabular} \\
\hline & 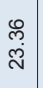 & 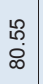 & $\begin{array}{l}\stackrel{M}{0} \\
\stackrel{g}{\sigma}\end{array}$ & 定 & $\begin{array}{l}\stackrel{\circ}{0} \\
\stackrel{i}{\sim}\end{array}$ & 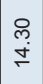 & & 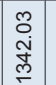 & $\begin{array}{l}\stackrel{0}{\grave{2}} \\
\stackrel{5}{\infty}\end{array}$ \\
\hline N & เ) & $m$ & $\infty$ & 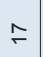 & $=$ & 0 & 0 & న్ & \\
\hline 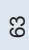 & $i$ & 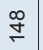 & 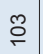 & o & q & N & $\stackrel{\infty}{\sim}$ & 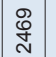 & \\
\hline N & 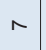 & $m$ & $\infty$ & $\stackrel{\sim}{\sim}$ & $\stackrel{\curvearrowleft}{\sim}$ & $\sim$ & 0 & $\stackrel{\text { S }}{\text { N }}$ & \\
\hline i & 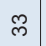 & ָָ & Бे & $\cong$ & $\stackrel{\mathscr{m}}{\infty}$ & ఇ & $\stackrel{\infty}{\sim}$ & $\frac{J}{\sim}$ & 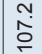 \\
\hline$\sim$ & の & $\sim$ & $\infty$ & $\cong$ & $\stackrel{\Xi}{ }$ & $\sim$ & 0 & $\mid \begin{array}{l}: \\
\stackrel{\infty}{*}\end{array}$ & $\stackrel{m}{\stackrel{m}{\rho}}$ \\
\hline is & $\bar{m}$ & 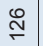 & $\Sigma$ & ల్ల & I & i & $\stackrel{\infty}{\simeq}$ & $\begin{array}{l}0 \\
\\
g \\
r\end{array}$ & $\stackrel{\circ}{\stackrel{\sigma}{0}}$ \\
\hline m & $n$ & $\sigma$ & $\infty$ & $\cong$ & $\stackrel{\Xi}{ }$ & N & $m$ & ते & ֶֻ. \\
\hline 足 & ㅇ & ㅇ & $\stackrel{8}{\circ}$ & ৪ & 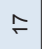 & $\stackrel{0}{\circ}$ & $\cong$ & $\mid$\begin{tabular}{l}
0 \\
$\stackrel{0}{0}$ \\
\hdashline
\end{tabular} & $\begin{array}{l}\infty \\
\infty \\
\infty\end{array}$ \\
\hline m & $n$ & 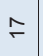 & の & $\stackrel{ \pm}{N}$ & $\cong$ & $\Sigma$ & $\underline{z}$ & : & लें \\
\hline$\approx$ & $\bar{N}$ & 同 & in & $\stackrel{\infty}{\sim}$ & ஓ & $\underline{s}$ & $\frac{\pi}{z}$ & 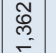 & $\begin{array}{l}\infty \\
\stackrel{+}{\dot{d}}\end{array}$ \\
\hline$\nabla$ & $\circ$ & $\stackrel{\circ}{\ulcorner}$ & $\simeq$ & $\stackrel{\sim}{\sim}$ & $\cong$ & - & $\sim$ & 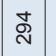 & f́ \\
\hline$\stackrel{9}{\leftarrow}$ & $\stackrel{\sim}{\sim}$ & $\stackrel{\circ}{\sim}$ & F & $\div$ & $\stackrel{\Xi}{\square}$ & $=$ & 0 & $\stackrel{\stackrel{\leftrightarrow}{\prime}}{\rightleftharpoons}$ & م̂. \\
\hline 0 & 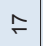 & 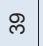 & の & $\bar{m}$ & $\stackrel{\circ}{\sim}$ & - & $\sim$ & $\underset{\substack{\infty \\
\infty}}{\mid}$ & \begin{tabular}{|l}
$\infty$ \\
$\infty$ \\
$\infty$
\end{tabular} \\
\hline$\stackrel{\infty}{\sim}$ & i & К & 8 & o & $\stackrel{\infty}{\sim}$ & \pm & ద & 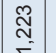 & $\frac{1}{\overline{0}}$ \\
\hline ص & $\stackrel{\infty}{\sim}$ & g & $\stackrel{2}{\circ}$ & প্) & $\stackrel{\llcorner}{\leftarrow}$ & - & - & $\begin{array}{l}\hat{\infty} \\
\text { d }\end{array}$ & $\stackrel{+}{\stackrel{\alpha}{\sigma}}$ \\
\hline$\stackrel{\overbrace{}}{\sim}$ & $\bar{N}$ & in & ले & $\infty$ & $\stackrel{\circ}{\circ}$ & $f$ & $m$ & E & $\mid \begin{array}{l}\infty \\
\infty \\
\stackrel{d}{\alpha}\end{array}$ \\
\hline$F$ & - & $\approx$ & $\stackrel{\infty}{\sim}$ & $\approx$ & $\stackrel{m}{\sim}$ & 0 & 0 & $\underset{\substack{\infty \\
\infty}}{\mid}$ & $\underset{\sigma}{\stackrel{\alpha}{\sigma}}$ \\
\hline$\stackrel{0}{\stackrel{0}{2}}$ & $\simeq$ & $\stackrel{\infty}{\sim}$ & $\stackrel{\text { N }}{ }$ & 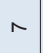 & ๑ & 0 & $\wedge$ & $\begin{array}{l}\hat{6} \\
\hat{0}\end{array}$ & 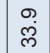 \\
\hline$\cong$ & 0 & $\simeq$ & $\bar{N}$ & の & 0 & 0 & 0 & 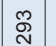 & $\stackrel{\jmath}{\dot{f}}$ \\
\hline$\cong$ & $\wedge$ & ల్m & r & - & $\cong$ & $\infty$ & $\sim$ & i & 卢 \\
\hline$\stackrel{m}{9}$ & 0 & $=$ & 0 & i & $\wedge$ & in & 0 & İ & 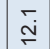 \\
\hline$\stackrel{\llcorner}{\leftarrow}$ & 0 & ஓ & 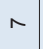 & $m$ & $\stackrel{\circ}{\circ}$ & 0 & $\sim$ & 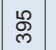 & $\stackrel{\infty}{\infty} \underset{\alpha}{\alpha}$ \\
\hline 产 & 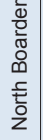 & & $\frac{\sqrt{\frac{c}{\tilde{w}}}}{\underline{\underline{w}}}$ & & 产 & $\mid \begin{array}{l}\vec{\sigma} \\
\vec{\sigma} \\
\frac{\vec{J}}{\sigma} \\
\frac{\sigma}{\alpha}\end{array}$ & 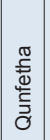 & $\mid \begin{array}{l}\vec{J} \\
5 \\
\stackrel{5}{\vdash}\end{array}$ & 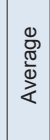 \\
\hline
\end{tabular}

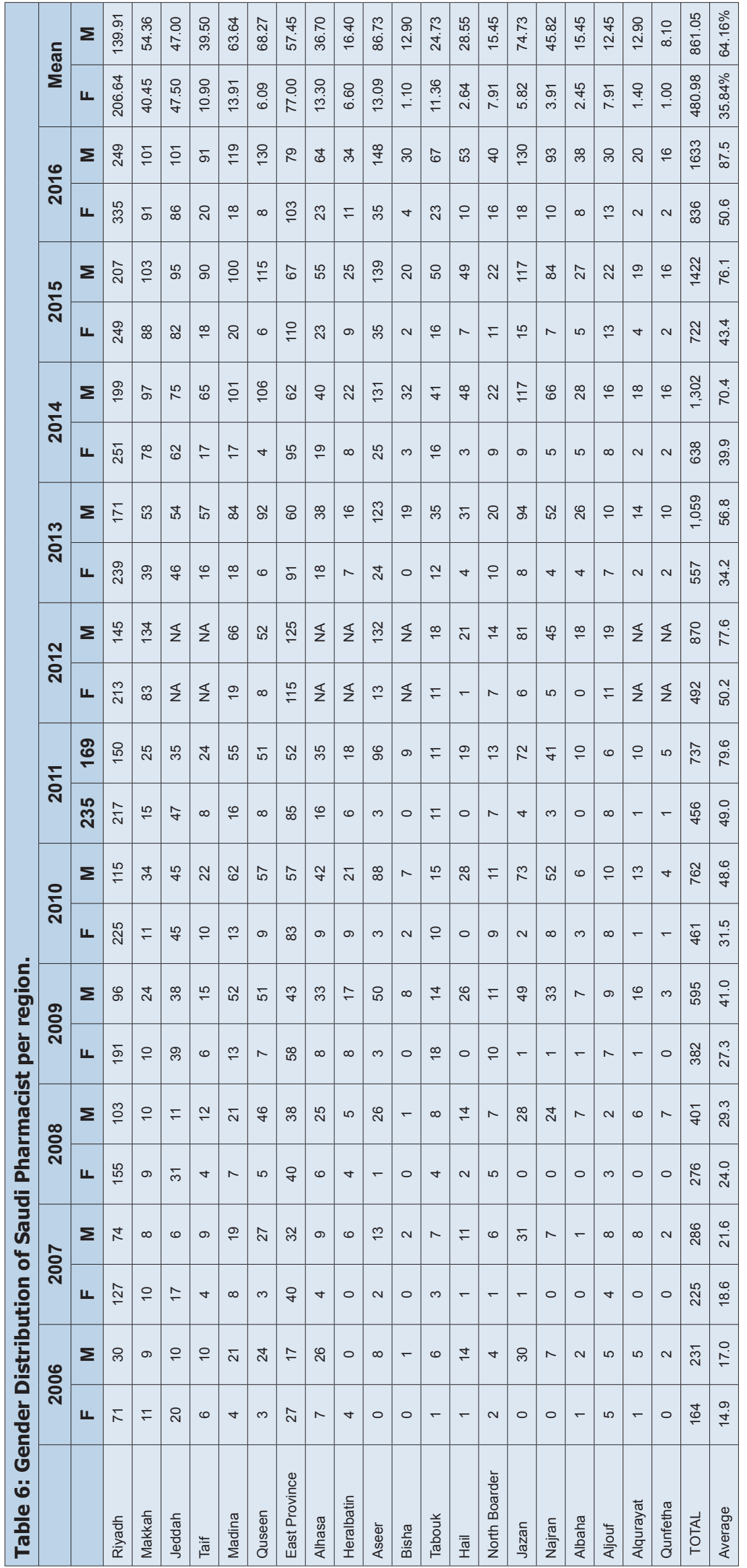


by Gaither, Caroline A., et al. and Gal, Diane et al..$^{[3,8-9]}$ The difference in the number of pharmacists between countries was very high, maybe because of the number of pharmacy colleges, and the importance and impact of the pharmacist were not apparent to the $\mathrm{MOH}$ administration. Also, most of the healthcare administrators thought that there is no difference between the pharmacist and pharmacy technician. The hospital pharmacists at $\mathrm{MOH}$ showed a significant impact on reducing medication errors, improving patient outcomes and avoiding millions of US dollar annually. ${ }^{[32-34]}$ The nationality results showed that most of the pharmacists were from Saudi due to the Saudisation plan of MOH. Another parameter of pharmacy workforces could not compare with studies because it missed at $\mathrm{MOH}$ statistical books. The number of the pharmacists should increase according to the updated guidelines of $\mathrm{MOH}$ pharmacy staff standards to provide full pharmaceutical care services at MOH hospital in Kingdom of Saudi Arabia.

\section{CONCLUSION}

Over the previous years, the pharmacist increased especially female pharmacist. The nationality of Saudi pharmacist increases and need more percentage to complete the MOH plan of Saudisation. The detail of pharmacy workforces missed due unreported at $\mathrm{MOH}$ statistical books. Pharmacy workforce's investigation everyone to two years is highly recommended at $\mathrm{MOH}$ hospitals in Kingdom of Saudi Arabia.

\section{ACKNOWLEDGEMENT}

None

\section{CONFLICT OF INTEREST}

None

\section{ABBREVIATION USED}

KSA: Kingdom of Saudi Arabia; MOH: Ministry of Health;

\section{REFERENCES}

1. ASHP Statement on Pharmaceutical Care. Am J Hosp Pharm. 1993;50:1720-3.

2. ASHP Guidelines on a Standardized Method for Pharmaceutical Care. Am Soc Heal Pharm. 1996;53(14):1713-6.

3. Mcleod DC. Contrast in Pharmacy Manpower Planning between the United States and Northen European Countries. Drug Intell Clin Pharm. 1986;20:210-2.

4. HRSA. A study of the supply and demand for pharmacists. Department of Health and Human Services. 2000;100. Available from: www.fip.org/humanresource.

5. Bond CA, Raehl CL, Patry R. Evidence-based core clinical pharmacy services in United States hospitals in 2020: services and staffing. Pharmacotherapy. 2004;24(4):427-40.

6. Bond CA, Raehl CL. Clinical pharmacy services, pharmacy staffing, and adverse drug reactions in United States hospitals. Pharmacotherapy. 2006;26(6):735-47.

7. Bond CA, Raehl CL. Clinical pharmacy services, pharmacy staffing, and hospital mortality rates. Pharmacotherapy. 2007;27(4):481-93.

8. Hawthorne N, Anderson C. The global pharmacy workforce: A systematic review of the literature. Hum Resour Health. 2009;7(1):48.

9. Gal D, Bates I. 2012. FIP Global Pharmacy Workforce Report. 2012. Available from: www.fip.org/humanresource

10. Workforce H. Pharmacist Workforce. 2012 - Provincial / Territorial Highlights. 2012.

11. Health Workforce Australia. Australia's Health Workforce Series: Pharmacists in Focus. 2014:1-47.
12. Gaither CA, Schommer JC, Doucette WR, Kreling DH, Mott DA. 2014 National Pharmacist Workforce Survey. Workforce. 2015. Available from: http://www. pharmacy.wsu.edu/information/2009.Pharmacist.Workforce.Survey.pdf.

13. Evan Robinson, Natalia Shcherbakova LB. Assessment of Pharmacy Manpower and Services in New England. Journal Pharm Pract. 2016;29(6):549-55.

14. Pedersen CA, Schneider PJ, Scheckelhoff DJ. ASHP national survey of pharmacy practice in hospital settings: Dispensing and administration - 2014. Am J Heal Pharm. 2015;72:1119-37.

15. Saudi Ministry of Health. Health Statistical Year Book 2006. Saudi Ministry of Health. 2006. Available from: http://www.moh.gov.sa/en/Ministry/Statistics/book/ Pages/default.aspx.

16. Health Statistical Year Book. Saudi Ministry of Health. 2007. Available from: http:// www.moh.gov.sa/en/Ministry/Statistics/book/Pages/default.aspx.

17. Health Statistical Year Book. Saudi Ministry of Health. 2008. Available from: http:// www.moh.gov.sa/en/Ministry/Statistics/book/Pages/default.aspx.

18. Health Statistical Year Book. Saudi Ministry of Health. 2009. Available from: http:// www.moh.gov.sa/en/Ministry/Statistics/book/Pages/default.aspx.

19. Health Statistical Year Book. Saudi Ministry of Health. 2010. Available from: http:// www.moh.gov.sa/en/Ministry/Statistics/book/Pages/default.aspx.

20. Health Statistical Year Book. Saudi Ministry of Health. 2011. Available from: http:// www.moh.gov.sa/en/Ministry/Statistics/book/Pages/default.aspx.

21. Health Statistical Year Book. Saudi Ministry of Health. 2012. Available from: http:// www.moh.gov.sa/en/Ministry/Statistics/book/Pages/default.aspx.

22. Health Statistical Year Book 2013. Saudi Ministry of Health. 2013. Available from: http://www.moh.gov.sa/en/Ministry/Statistics/book/Pages/default.aspx.

23. Health Statistical Year Book. Saudi Ministry of Health. 2014. Available from: http:// www.moh.gov.sa/en/Ministry/Statistics/book/Pages/default.aspx.

24. Health Statistical Year Book. Saudi Ministry of Health. 2015. Available from: http:// www.moh.gov.sa/en/Ministry/Statistics/book/Pages/default.aspx.

25. Health Statistical Year Book. Saudi Ministry of Health. 2016. Available from: http:// www.moh.gov.sa/en/Ministry/Statistics/book/Pages/default.aspx.

26. Medication Management (MM). In: National Hospital Standards. 2nd Editio. Saudi Central Board for Accreditation of Healthcare Institutions. 2015. p. 194-211. Available from: http://www.cbahi.gov.sa.

27. The Joint Commission. Comprehensive Accreditation Manuals. Joint Commission Resources. Available from: http://www.jcrinc.com/store/publications/manuals/.

28. Alomi YA, Alghamdi SJ, Alattyh RA. Strategic Plan of General Administration of Pharmaceutical Care at Ministry of Health in Saudi Arabia 2012-2022. J Pharm Pharm Scien. 2015;1(3):1-8.

29. Ministry of Health. A Cheivements. 2013. 1st Ed. Riyadh, Saudi Arabia: Ministry of Health, Saudi Arabia. 2011. 132-135 p.

30. Alomi YA, Ahmed Alomi Y, Pharm B, Clin Pharm M. A new Guidelines on Hospital Pharmacy Manpower in Saudi Arabia. J Pharm Pract Community Med. 2016;2(2).

31. Alomi YA. Primary Care Center Pharmacy Manpower New Guidelines in Saudi Arabia. J Pharmacol Clin Res. 2016;1(1).

32. Alomi YA, Aldosori N, Alhadab M, Alotaibi NR, Shubbar NA, Jadkarim MM, et al. Impact of Clinical Pharmacist Consultation Visits at Ministry of Health Hospitals in Saudi Arabia: Clinical Pharmacy Services and Pharmacy Workforce. J Pharm Pract Community Med. 2017;3(3).

33. Alomi YA, Aldosori N, Alhadab M, Alotaibi NR, Al-Shubbar N, Alotaibi T, et al. The Value of Clinical Pharmacist Consultation Visits at Ministry of Health Hospitals in Saudi Arabia: Intravenous Admixture Services and Pharmacy Total Quality Management. J Pharm Pract Community Med. 2017;3(3).

34. Alomi YA, Almudaiheem HY. Clinical And Economic Outcomes Of Pharmacist Intervention During Therapeutic Drug Monitoring Program In Saudi Arabia. Value Heal. 2016;19(7):A465-6. 